\title{
ÉVOLUTION DES EMISSIONS CORONALES AU COURS DE LA VIE D'UN CENTRE ACTIF
}

\author{
J. L. LeRoY, J. RösCh, M. TRellis \\ (Observatoire du Pic-du-Midi et Observatoire de Nice, France)
}

\begin{abstract}
A BSTRACT
A study of the development in the solar corona of active centers born during the CSSAR period leads to the following remarks: the 'enhancement' of coronal emissions seems to take place first within a localized 'core' close to the plage, then to extend to a much larger 'halo'; the core from which all the radiations under study originate does not last much more than the spots, whereas the 'halo' is characterized by the major importance of the emission at $5303 \AA$, and lasts as long as the $\mathrm{K} 3$ plages. These features can be explained by the assumption that the enhancement is inhomogeneous during the first part of its life, then becomes homogeneous after the core has disappeared.
\end{abstract}

La Coopération pour l'étude des régions solaires actives (CSSAR, 10 mars 30 octobre 1965) a présenté un grand intérêt pour la compréhension des phénomènes coronaux. En effet, les observations des émissions coronales sont généralement effectuées au limbe et doivent être réalisées de préférence pendant des périodes de faible activité solaire si l'on veut que les phénomènes associés à différents centres actifs puissent être observés séparément. Mettant à profit le fait qu'un très grand nombre de données étaient disponibles, nous avons étudié le développement dans la couronne des centres actifs apparus pendant la période 'CSSAR' à l'aide des observations optiques et radioélectriques qui avaient été recueillies notamment:

(1) Les intensités des raies d'émission de FexIV à $5303 \AA$ et de Fex à $6374 \AA$.

(2) Les mesures de la brillance de la couronne blanche déduites d'observations polarimétriques qui fournissent la grandeur du produit $p B(p=$ proportion de lumière polarisée, $B=$ brillance de la couronne) moyennant certaines corrections.

Ces deux catégories d'observations sont disponibles de façon presque quotidienne pour la basse couronne (altitude de $40000 \mathrm{~km}$ environ). On peut admettre qu'elles ont une résolution dans l'espace de l'ordre de 1.5 et une résolution dans le temps de 24 heures.

(3) Les radiohéliogrammes obtenus tous les jours à Stanford sur $9 \cdot 1 \mathrm{~cm}$ et à Fleurs sur $21 \mathrm{~cm}$ avec une résolution de $3^{\prime}$ environ.

La réduction à une même échelle d'intensité des différentes mesures des raies 5303 et $6374 \AA$ a été effectuée à l'Observatoire du Pic-du-Midi: ce travail a été mené de façon empirique en comparant entre elles les couronnes semestrielles moyennes obtenues par chacun des observatoires participant à la Coopération. Dans la suite 
de cet exposé, les intensités seront donc exprimées dans une seule échelle qui est celle du Pic-du-Midi.

Pour la couronne blanche, l'échelle adoptée est celle de Haleakala dont les observations étaient de beaucoup les plus nombreuses. Les cartes ont bénéficié de quelques observations de Norikura et du Pic-du-Midi qui ont été ajustées de façon très approximative à l'échelle de Haleakala.

Les radiohéliogrammes ont été relevés dans le bulletin des Solar Geophysical Data (CRPL.F., Part B). Aucun problème d'homogénéité des mesures ne se posait puisque toutes les observations proviennent d'un seul observatoire pour une longueur d'onde donnée.

Enfin, les données relatives aux centres actifs photosphériques et chromosphériques ont été déduites des spectrohéliogrammes $\mathrm{K} 1$ et $\mathrm{K} 3$ de l'Observatoire de Meudon et des cartes chromosphériques du même Observatoire.

Dans la suite de ce travail, nous suivrons la terminologie proposée par Billings (1966) et nous désignerons du nom de renforcement coronal ('enhancement' de Billings) l'ensemble des régions de la couronne qui sont perturbées par un centre actif. On réservera le terme de condensation aux régions les plus concentrées du renforcement coronal qui apparaissent parfois de façon éphémère quand le centre actif est au maximum de son développement.

\section{Présentation des cartes synoptiques}

Les observations disponibles n'ont pas été suffisamment continues pour que l'on puisse suivre sans interruption l'évolution de tous les centres actifs de leur naissance jusqu'à leur disparition. Nous illustrerons donc l'évolution de ce que nous considérons comme un centre actif moyen en présentant des exemples relatifs à des centres actifs différents, en l'occurence ceux dont le stade d'existence considéré a été le mieux observé.

Les illustrations qui constituent ce paragraphe sont des cartes synoptiques dressées à partir des observations au bord; elles tiennent donc "autant du film que de la carte", (Trellis, 1957). Ce mode de présentation est imposé dans le cas de la couronne blanche et des radiations 5303 et $6374 \AA$; nous l'avons également adopté pour les émissions radioélectriques, bien que dans ce cas de véritables cartes soient également disponibles, pensant qu'il valait mieux comparer entre elles des cartes synoptiques qui présentent - sinon les mêmes - du moins des altérations analogues dûes à la perspective. Il résulte de ce choix que les cartes synoptiques des émissions radioélectriques sont sans doute imprécises à cause des effets de réfraction notamment.

Outre les données relatives à la couronne blanche et aux émissions à 5303 et $6374 \AA$, nous présentons sur les Figures 1 à 4 des cartes synoptiques des émissions à $9 \cdot 1 \mathrm{~cm}$; nous les avons préférées aux observations à $21 \mathrm{~cm}$, bien que ce soient ces dernières qui soient observées en principe à la même altitude que les radiations visibles étudiées, 


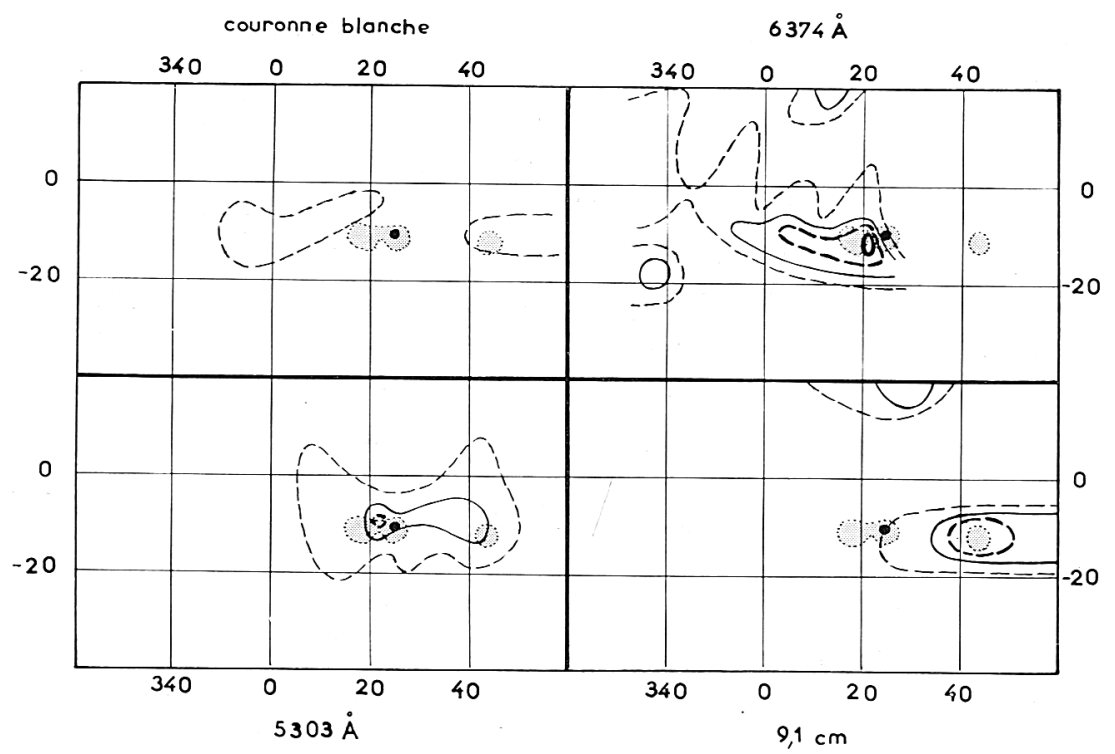

FIG. 1. Cartes synoptiques établies à partir des observations effectuées au bord ouest du 8 au 14 juin 1965, 10 jours après la naissance de CSSAR no. 5. La signification des isophotes est précisée dans l'annexe aux figures.

parce que les cartes que nous avons établies ont montré que l'apparition d'un centre actif au bord solaire est beaucoup plus sensible à $9 \mathrm{~cm}$ qu'à $21 \mathrm{~cm}$.

\section{Evolution du renforcement coronal}

Nous avons dressé des graphiques qui donnent la variation au cours du temps de l'intensité maximale observée, pour chaque type de rayonnement étudié, à proximité de la plage faculaire (de façon plus précise, l'intensité maximale obtenue, après lissage des mesures de diverses provenances, dans un rayon de $10^{\circ}$ héliographiques autour du centre de gravité des plages faculaires K 3). Nous avons porté en ordonnée l'intensité maximale plutôt que l'étendue du renforcement coronal parce que les altérations dûes aux effets de perspective doivent être beaucoup plus réduites pour cette variable.

La Figure 5 donne ces graphiques sur lesquels on remarque tout de suite une très grande dispersion des points dûe en partie aux deux raisons suivantes: (1) L'âge des centres actifs est mal déterminé dans le cas des centres nés sur l'hémisphère invisible du Soleil. (2) Il n'est pas absolument légitime de mélanger les observations provenant de différents centres actifs puisque l'on sait par ailleurs dans le cas des émissions à $5303 \AA$ que la durée de vie n'est pas indépendante de l'intensité maximale atteinte (Trellis, 1959). Pour limiter la dispersion dûe à cette dernière cause nous avons exclu 


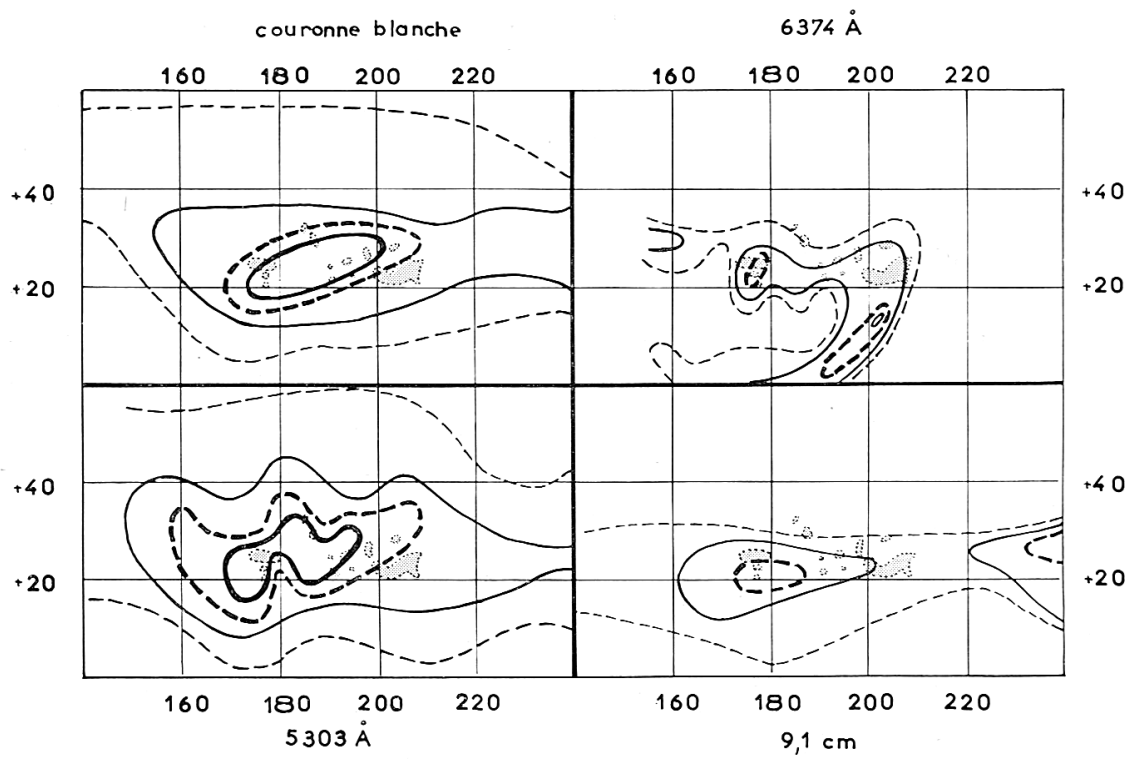

Fig. 2. Cartes synoptiques établies à partir des observations effectuées au bord ouest du 9 au 16 octobre 1965, 20 jours environ après la naissance de CSSAR no. 9 (à droite). Au centre gauche des cartes on voit les restes de CSSAR no. 8 âgé de 40 jours. La signification des isophotes est précisée dans l'annexe aux figures.

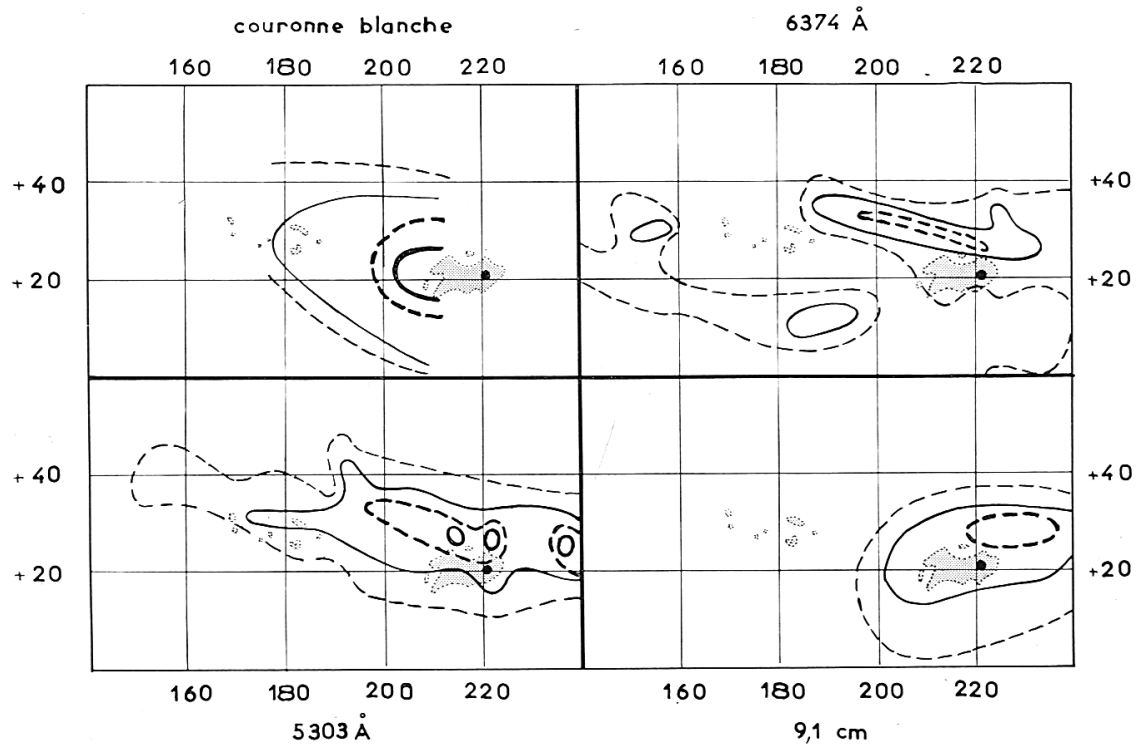

FIg. 3. Cartes synoptiques établies à partir des observations effectuées au bord est du 8 au 15 juin 1965, 35 jours environ après la naissance de CSSAR no. 3 (à droite) et no. 4 (à gauche). La signification des isophotes est précisée dans l'annexe aux figures. 


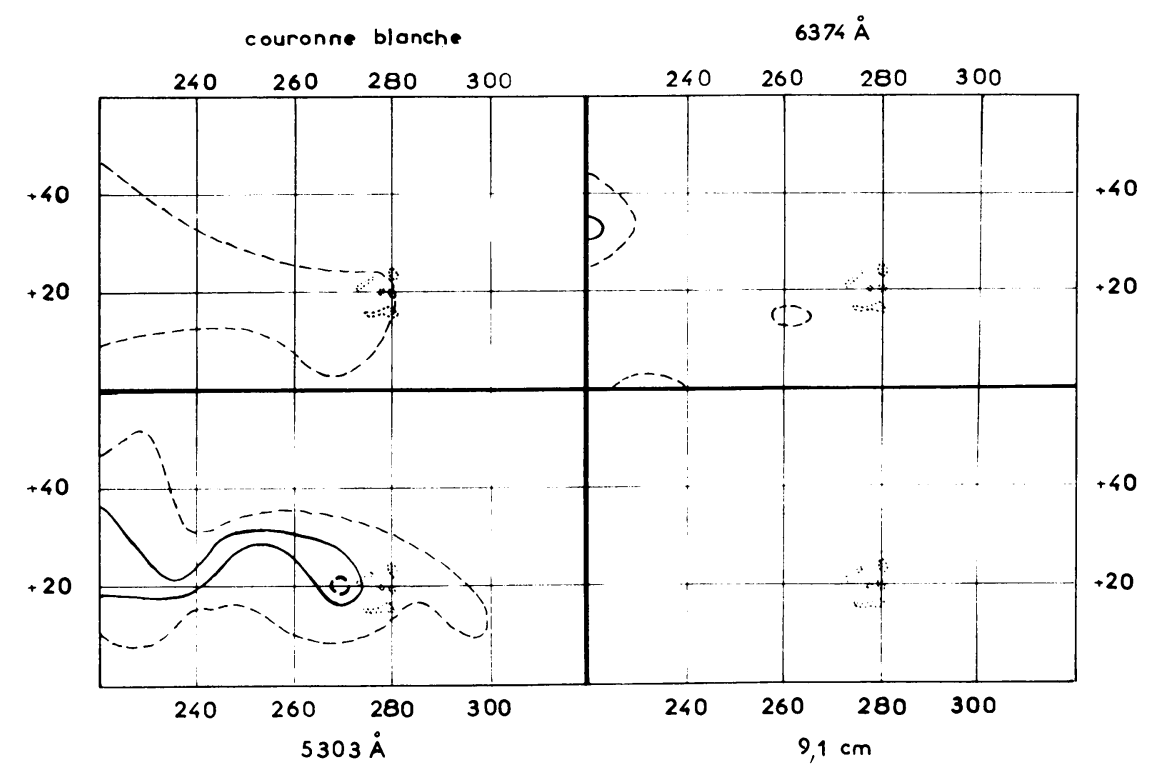

Fig. 4. Cartes synoptiques établies à partir des observations effectuées au bord est du 25 juillet au 2 août 1965, 25 jours après la naissance de CSSAR no. 7. La signification des isophotes est précisée dans l'annexe aux figures.

de nos graphiques les points correspondant aux centres CSSAR no. 3, 4, 8, et 9, qui semblaient les plus intenses et d'ailleurs étaient partiellement mélangés. Il faut noter à ce propos que par suite de la superposition de centres actifs parasites la vie des centres retenus n'a pas été suivie en entier sauf pour CSSAR no. 5; dans six autres cas on a pris en considération une partie seulement, atteignant au moins 30 jours, de l'existence complète du centre.

Ceci étant, on s'aperçoit, malgré la dispersion des points, que les courbes de la Figure 5 se classent en deux familles: d'une part le renforcement coronal en lumière blanche et à $5303 \AA$ qui a une durée de vie longue, comparable à celle des facules. D'autre part les émissions à $6374 \AA$ et à $9 \cdot 1 \mathrm{~cm}$ qui ont une durée de vie courte, comparable à celle des taches. On est donc d'abord amené à penser que l'évolution coronale d'un centre actif comprend deux étapes bien différentes.

On peut noter ensuite (1) que l'énorme contraste des émissions à $5303 \AA$ pendant toute la vie d'un centre actif confirme le résultat établi précédemment (Trellis, 1959), qui donne la radiation $5303 \AA$ comme un indicateur extraordinairement sensible de l'activité solaire, (2) que la même propriété apparaît, un peu moins accusée, pour la radiation $6374 \AA$, mais seulement pendant le début de la vie d'un centre actif; on pourrait dire que la radiation $6374 \AA$ est un bon indicateur des centres actifs jeunes, (3) que bien que le renforcement coronal observé en lumière blanche ait à peu près la même durée de vie que les émissions à $5303 \AA$, son faible contraste en fait un indice 

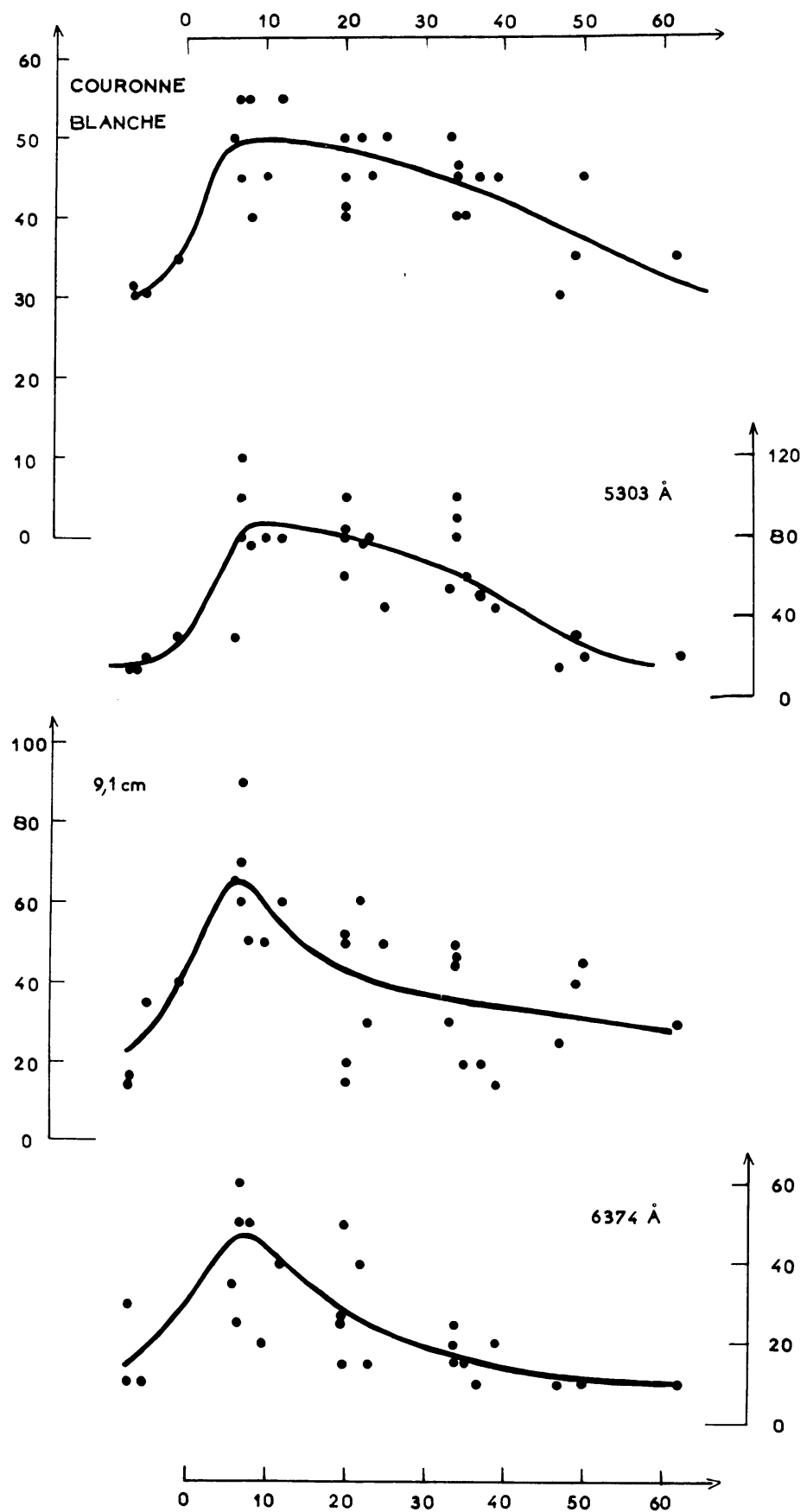

FIG. 5. Evolution de l'intensité des émissions coronales associées à un centre actif en fonction du temps. On a porté en abscisse l'âge des centres actifs compté en jours et en ordonnée l'intensité des diverses émissions considérées exprimées dans les unités qui sont explicitées dans l'annexe aux figures. 
d'activité beaucoup moins sensible notamment pendant le déclin d'un centre actif; on notera en particulier que l'augmentation relative de l'intensité de la couronne blanche, au moment du plus grand développement du centre actif, est beaucoup plus petite que celle des radiations $5303 \AA, 6374 \AA$ et $9 \cdot 1 \mathrm{~cm}$.

On constatera par ailleurs que la partie des courbes de la Figure 5 qui est relative à la naissance des centres actifs est très imprécise et l'on se gardera donc d'en tirer quelque interprétation concernant cette phase de leur vie.

Nous remarquerons enfin que la résolution dans le temps des observations qui viennent d'être analysées est de 1 jour environ, c'est-à-dire qu'elle est grande devant la durée de vie des condensations qui peuvent apparaître au sein du renforcement coronal quand le centre actif est à son paroxysme. On doit donc considérer que les condensations coronales échappent à notre étude.

\section{Interprétation de l'évolution observée}

Les deux formes d'évolution mises en évidence par la Figure 5 et les différentes propriétés des cartes (Figures 1 à 4) nous conduisent à l'interprétation suivante:

Pendant une première phase de la vie d'un centre actif, que nous appellerons phase $A$ et qui correspond à peu près à la période d'existence des taches, le renforcement coronal est essentiellement hétérogène en densité et en température. Cela explique (1) que pour une augmentation modérée de la densité électronique moyenne (cf. le faible contraste de la couronne blanche) les émissions à $5303 \AA, 6374 \AA$ et $9 \cdot 1 \mathrm{~cm}$ puissent devenir très intenses, vraisemblablement dans des régions où $\mathrm{Ne}^{2}$ est localement très grand, (2) que des émissions qui correspondent à des conditions d'excitation très différentes (6374 Å et $5694 \AA$ par exemple) soient simultanément intenses (Nishi et Nakagomi, 1963).

Nous remarquons que cette phase $A$ cesse peu après la disparition des taches ce qui permettrait de penser que le champ magnétique qui est associé à ces dernières joue un rôle important dans l'établissement et le maintien des hétérogénéités qui sont envisagées ici. On se rappelle d'autre part que les jets coronaux intenses s'observent le plus souvent à l'est et à l'ouest des facules (Trellis, 1957), c'est-à-dire précisément à proximité du lieu d'apparition des taches ce qui renforce l'idée que ces dernières, ou leur champ magnétique, jouent peut être un rôle déterminant pendant cette première phase de la vie du renforcement coronal.

Pendant la deuxième phase (phase $B$ ), qui s'étend entre la disparition de taches et celle des facules, les hétérogénéités disparaissent et avec elles les régions où $\mathrm{Ne}^{2}$ est très grand. La température du renforcement coronal s'uniformise à une valeur favorable à l'excitation de la raie $5303 \AA$. Ceci explique (1) la disparition des émissions à $6374 \AA$, (2) l'affaiblissement des émissions à $9 \cdot 1 \mathrm{~cm}$, (3) la persistance très importante des émissions intenses à $5303 \AA$, et (4) la ressemblance entre les isophotes de la couronne blanche et celles de la radiation $5303 \AA$. 
Ces deux grandes phases de la vie d'un renforcement coronal peuvent encore être divisées en plusieurs périodes, dans lesquelles on retrouve les propriétés aperçues sur les Figures 1 à 4 et également les caractéristiques de plusieurs modèles classiques de renforcement coronaux.

Au début $A 1$ de la phase $A$, toutes les émissions optiques et radioélectriques apparaissent ensemble dans un noyau bien concentré à proximité immédiate de la facule (cet exemple est à peu près illustré par la Figure 1). On peut penser que ce noyau marque la limite des régions où existent de fortes densités électroniques et des températures non-uniformes.

La fin $A 2$ de la phase $A$ correspond au maximum d'activité du centre actif. (Figure 2). On peut alors distinguer: (1) Un noyau central hétérogène qui fait suite au noyau de la phase $A 1$ où apparaissent simultanément les émissions à $5303 \AA, 6374 \AA$ et $9 \cdot 1 \mathrm{~cm}$. (2) Un halo périphérique (Waldmeier, 1963), qui peut être homogène et à une température relativement élevée et où l'on observe principalement une grande extension des émissions à $5303 \AA$. Cette structure doit entraîner que les maximums d'émission à $5694 \AA, 6374 \AA$ et $9 \cdot 1 \mathrm{~cm}$ sont situés dans le noyau central à proximité immédiate de la facule alors que les maximums à $5303 \AA$ peuvent se situer plus loin en bordure de cette dernière. Telle est bien la situation moyenne (non réalisée sur la Figure 2 mais visible dans le cas de CSSAR no. 4 sur la partie gauche de la Figure 3 ) décrite par de nombreux auteurs (Billings, 1966) pour la radiation jaune et par Trellis (1957) pour les maximums verts et pour certains maximums rouges.

Le début $B 1$ de la phase $B$ est marqué par la disparition progressive du noyau hétérogène qui entraîne l'affaiblissement des émissions à $6374 \AA$ et à $9 \cdot 1 \mathrm{~cm}$ auprès des facules. Par contre le halo se développe et avec lui les émissions à $5303 \AA$ qu'il favorise. De plus, sa bordure extérieure est parfois marquée par des émissions à $6374 \AA$, dont l'existence avait déjà été signalée par Trellis (1957), qui doivent jalonner les régions où l'accroissement de densité électronique a précédé l'élévation de température. Le centre CSSAR no. 3, dans la partie droite de la Figure 3, illustre assez bien cette phase.

A la fin $B 2$ de la période $B$ les hétérogénéités ont complètement disparu et la température s'est uniformisée à une valeur favorable à l'émission à $5303 \AA$, qui restera donc seule intense notamment là où la densité est un peu plus élevée (Figure 4).

\section{Conclusion}

Il convient, pour terminer, de rappeler que l'interprétation précédente est fondée sur l'étude d'une douzaine de centres actifs seulement ; c'est dire qu'il faut la considérer comme un modèle de travail susceptible d'être largement amélioré ou modifié dans l'avenir.

Nous avons signalé à plusieurs reprises au cours de cette étude les limitations qui étaient introduites par le manque de pouvoir séparateur dans l'espace et dans le temps 
des observations de routine qui ont servi de base à notre travail. Une telle limitation est particulièrement regrettable quand on est amené à introduire la conception d'un noyau hétérogène pour expliquer les observations. Sans préjuger des progrès instrumentaux qui pourront marquer, d'ici le prochain minimum solaire, les observations de la couronne blanche, celles de l'ultra violet coronal et celle des émissions radioélectriques, on peut déjà remarquer que l'usage extensif de filtres monochromatiques, qui permettent d'obtenir des images détaillées des structures coronales dans la lumière de diverses radiations visibles, serait sans doute d'un grand secours pour une meilleure compréhension des phénomènes coronaux associés aux centres actifs.

\section{Annexe aux figures}

Les unités utilisées sont:

Pour les émissions à $5303 \AA$ et à $6374 \AA$ le millionième de la brillance de $1 \AA$ du spectre du centre du Soleil au voisinage de la longueur d'onde étudiée.

Pour la couronne blanche le cent millionième de la brillance du centre du Soleil dans l'intervalle spectral considéré.

Pour le rayonnement à $9 \cdot 1 \mathrm{~cm}$, une température de brillance de $1000^{\circ} \mathrm{K}$.

Sur les cartes des Figures 1 à 4 les isophotes sont dessinées successivement en tirets fins, en trait continu fin, en tirets épais et en trait continu épais quand on va des petites aux grandes intensités.

La représentation utilise quatre isophotes, qui correspondent aux intensités suivantes:

Couronne blanche: $p B=40-50-60-70$; unité $10^{-8} B_{\odot}$ dans l'échelle d'Haleakala.

Radiation $5303 \AA$ : $B=25-50-75-100$; unité $10^{-6} B_{\odot}$ dans l'échelle du Pic-du-Midi.

Radiation $6374 \AA$ : $B=20-30-40-50$; unité $10^{-6} B_{\odot}$ dans l'échelle du Pic-du-Midi.

Rayonnement à $9 \cdot 1 \mathrm{~cm}: T=40-60-80-100$; unité $10^{3}{ }^{\circ} \mathrm{K}$ dans l'échelle de Stanford.

Les plages faculaires chromosphériques sont indiquées par une zone grise délimitée par une ligne en pointillés et les taches sont figurées par un point noir.

\section{Bibliographie}

Billings, D.E. (1966) A Guide to the Solar Corona, Academic Press, New York.

Nishi, K., Nakagomi, Y. (1963) Publ. astr. Soc. Japan, 15, 56.

Trellis, M. (1957) Suppl. Ann. Astrophys., 5.

Trellis, M. (1959) Ann. Astrophys., 22, 845.

Waldmeier, M. (1963) Z. Astrophys., 58, 57.

\section{DISCUSSION}

Nussbaumer: You spoke of a strong enhancement in both the green and the red lines with only a slight increase in the mean electron density. Could you tell us to which increase in inhomogeneity one would have to conclude from these observations?

Rösch: I am sorry but I have no figures to answer that question. 
Fokker: A possibility to check the hypothesis, that coronal structure becomes more homogeneous as the active centre ages, may be the measurement of angular diameters of radio type-I stormbursts. The angular diameter is largely due to scattering on coronal irregularities. If coronal structure, late in the centre of activity's lifetime, were more smooth, angular diameters of stormbursts may be expected to decrease.

Rösch: This is an interesting possibility.

Elske Smith: I would like to point out the similarities of these observations with the extreme ultraviolet data which will be reported on by Dr. Neupert this afternoon. The enhancements of the XUV permitted lines are similar to that shown by the visible and radio data, and therefore fit fairly well with the model of inhomogeneous structures in the corona as suggested by Leroy and Trellis. I am, however, surprised at the rapid decay of the red line in comparison with the green line. This is divergent from the changes in the lines of different ionization in the ultraviolet. Perhaps, as you say, this may be attributed to a more rapid change in density than in temperature. 